\title{
A Survey on Internet of Things (IoT) based Smart Systems
}

\author{
Prof. Sathish, \\ Department of EEE, \\ Eritrea Institute of Technology, \\ Eritrea. \\ Email: sathesh4you@gmail.com
}

\author{
Dr. S. Smys, \\ Professor, Department of Computer Science and Engineering, \\ RVS Technical Campus, \\ Coimbatore, India. \\ Email:smys375@gmail.com
}

\begin{abstract}
Internet of Things (IoT) is a collection of networks, objects, devices that are connected using the Internet. It interacts with the environment both internally and externally. IoT senses the environment and acts accordingly. It gives the environment with advanced techniques and thus improves the quality of mankind. IoT allows the devices to communicate with each other either physically or virtually. With the help of IoT, the environment becomes smarter and can be connected with any device at any time. Nowadays people want to communicate with all the non-living things via the Internet. IoT is used to collect and analyze the data from various actuators and sensors then it is passed it over smartphones or computers through a wireless connection. There evolve the applications that use IoT and in each of the applications IoT plays a vital role in enhancing the living standards which includes home appliances, Smart energy, environment, and industries, etc. and makes a friendly environment. To make it more professional, IoT is used in the supply chain, transportation, logistics, automation, and remote monitoring. IoT delivers a change in the quality of individual life and it is widely distributed among the network of devices and provides a new ecosystem for the development of applications by understanding the development of the market in the early stages. IoT has the capabilities of delivering the services and demands of the people in this fast-moving world. This paper presents the survey of IoT smart systems applications and usages of the IoT environment.
\end{abstract}

Keywords: Internet of Things (IoT), Smart Home, Smart Cities, Smart System, Networks

\section{Introduction}

The Internet of Things (IoT) has the ability and flexibility to adapt to the environment easily. It makes the environment intelligent with the applications used on them (Wang et al. 2013). The IoT is better than the Machineto-Machine communications, GSM, GPS, Microcontroller, Microprocessor, GPRS, $2 \mathrm{G} / 3 \mathrm{G} / 4 \mathrm{G}$ networks. IoT is a combination of both hardware and software (Zhai et al. 2012). The main aim of IoT is to enable the device and to be connected all the time anywhere using a network (Soliman et al. 2013). IoT is evolved from machine-tomachine communication (M2M). In M2M communication, the devices are connected to the cloud and manage the collected data but in IoT, millions of smart devices, sensor nodes, and applications generate and share the data in real-time to decide on a time. Therefore, M2M paves the way for connectivity to IoT (Singh et al. 2014). The IoT architecture consists of three layers such as things, gateway, and cloud that comprise sensors, devices, and objects. Figure 1 shows the IoT architecture. The gateway consists of IoT protocols such as Bluetooth and ZigBee. Cloud is responsible for wireless communications such as cellular and Wi-Fi. Gateway and cloud are composed of Edge Computing. 
Journal of ISMAC (2020)

Vol.02/ No.04

Pages: 181-189

http://irojournals.com/iroismac/

DOI: https://doi.org/10.36548/jismac.2020.4.001

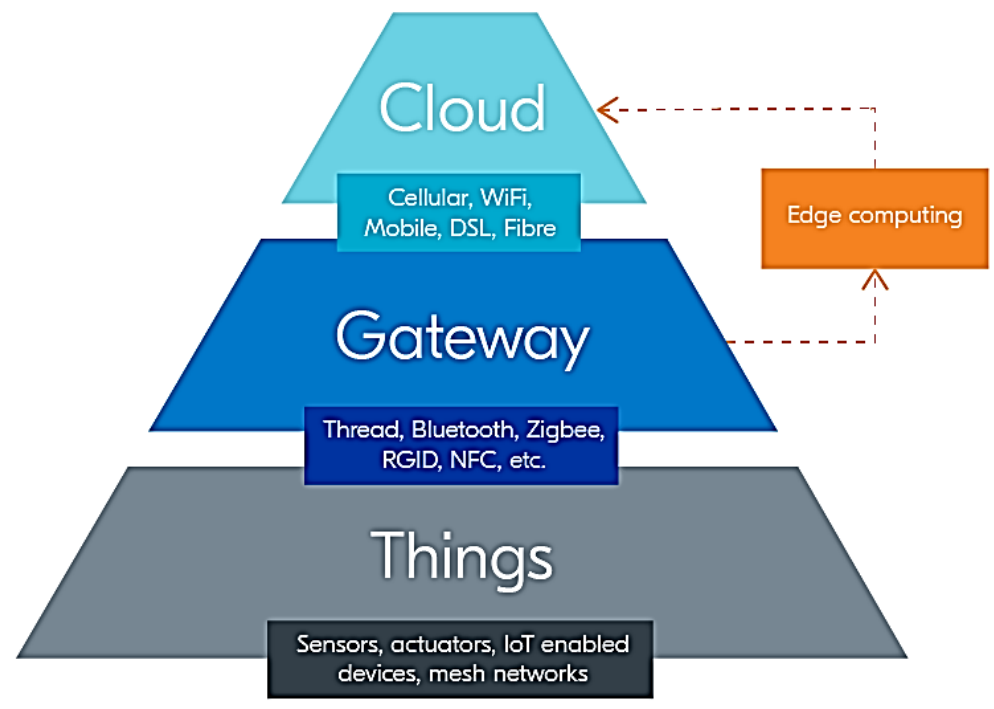

Fig.1 IoT Architecture

Internet of Things (IoT) connects the devices, objects through the internet using wireless technology. IoT helps to transfer, communicate, and share the data anywhere at any time via the internet (Yashiro et al. 2013). It creates a remote environment for accessing the data and it has been used in many real-time applications such as smart cities, smart homes, smart energy, smart agriculture, smart industry, and smart living. IoT has its characteristics which include interconnectivity, safety, heterogeneity, enormous scale, dynamic changes, and connectivity. The IoT environment is specified in figure 2. IoT is a combination of several technologies such as Embedded systems, pervasive computing, Actuators, Ambient Intelligence, Sensors, Internet Technologies and communication technologies, etc., IoT is classified based on their functionalities (Rathore et al. 2017). It consists of three functionalities there are Things oriented, Semantic oriented, and Internet-oriented. The main aim of IoT is to provide ease of operations, remote access control, configuration, and end-user. IoT provides seamless connectivity besides the heterogeneous networks.

Internet of Things is analogous with ploys and methodologies which restrain intelligent machines communicating with various objects, constraints, modules, and bases, and also sensors set out revolution will mount to address its troubles (Vandana et al. 2016). An immense amount of information has been created, handled for useful activities that drives life more protected and less extracting. The problem needs laborers who are devoted to that specific condition and should have step forward data consider the proffer's attention and the alliance. We can visualize enormous shrewd gadgets in our environment is because of IoT. 
Journal of ISMAC (2020)

Vol.02/ No.04

Pages: $181-189$

http://irojournals.com/iroismac/

DOI: https://doi.org/10.36548/jismac.2020.4.001

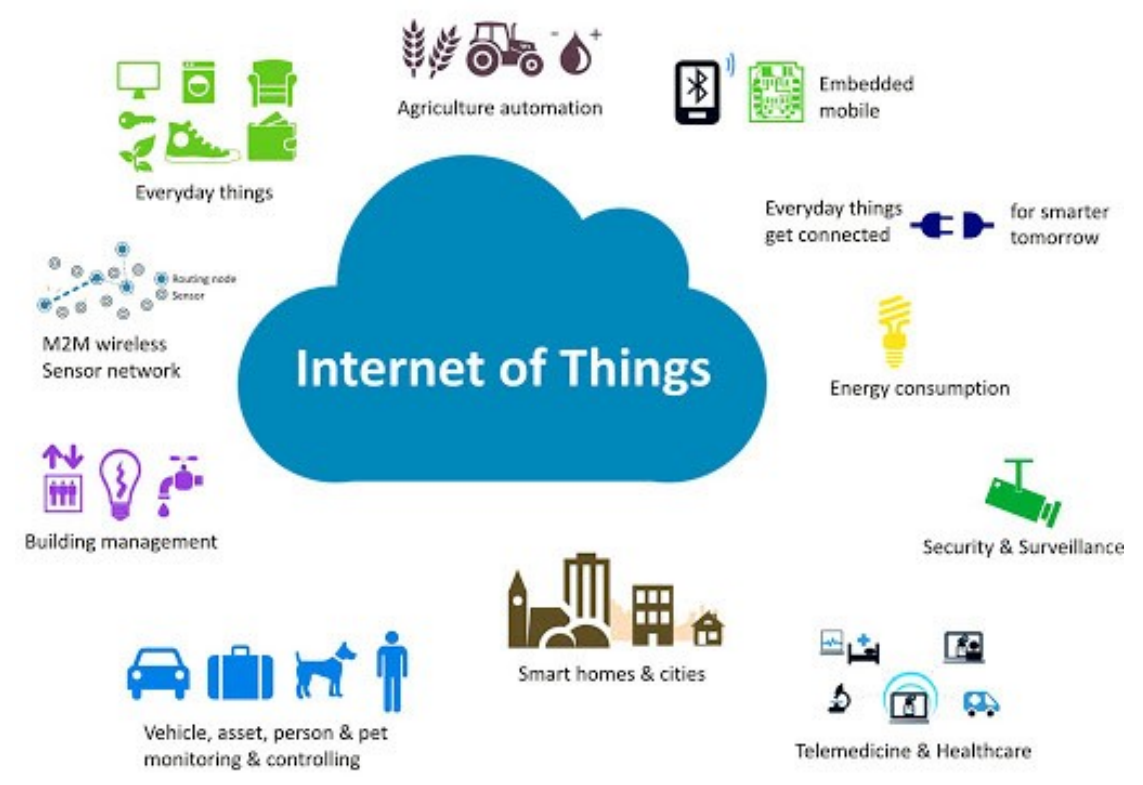

Fig.2 Overview of IoT Environment

Different individuals look towards areas of urban and relative work been carried out by them but the world will more confer with determining and activation for the things installed (Bhavna et al. ). Now a day's Mobile systems convey the network which can improvise the latest administrations and entreaty. In the availability of new technological applications workstations, autos, substructures, vivid meters, controlling pursuits and even with interfacing plays major roles. The sensor system idea is innovated by amalgamating mechanical frameworks of microelectronics, remote comparability.

Firstly, the applications of the sensor and detection process are probed and the survey strands influencing the contour of sensor arrangement are stated (Sharma et al. 2016). The structure is deliberated to process ably without human intervention if any need they can request to the framework through messages which will in turn respond with the information needed by the client. More investigation learnings are added to the IoT day by day. The affairs related to the interconnection of terminals and examining center, declining the terminals and referring the progress of transmission under the condition of the remote site, spectacular ambiance, and other biotic concerns (Patel et al. 2016). To improve the security skeleton this proposed system can be employed as segments, make responsiveness to the calamity situations, and get away from countless risks. To proceed with the business towards the market refinement, an intentional struggle is required which are driven by various opportunities. This market system has specified ascribes in the unions of executive dissemination, business models, capabilities to lease the administration of IoT and contrasting appeals will enrobe transferable systems.

\section{Applications of IoT}

IoT is implemented in most of the areas in day-to-day applications which covers lifestyle, retail, city, building, transportation, agriculture, healthcare, environment, and energy (Lee et al. 2015). Some of the applications are smart homes, smart cities, smart energy, and smart industry, etc. 


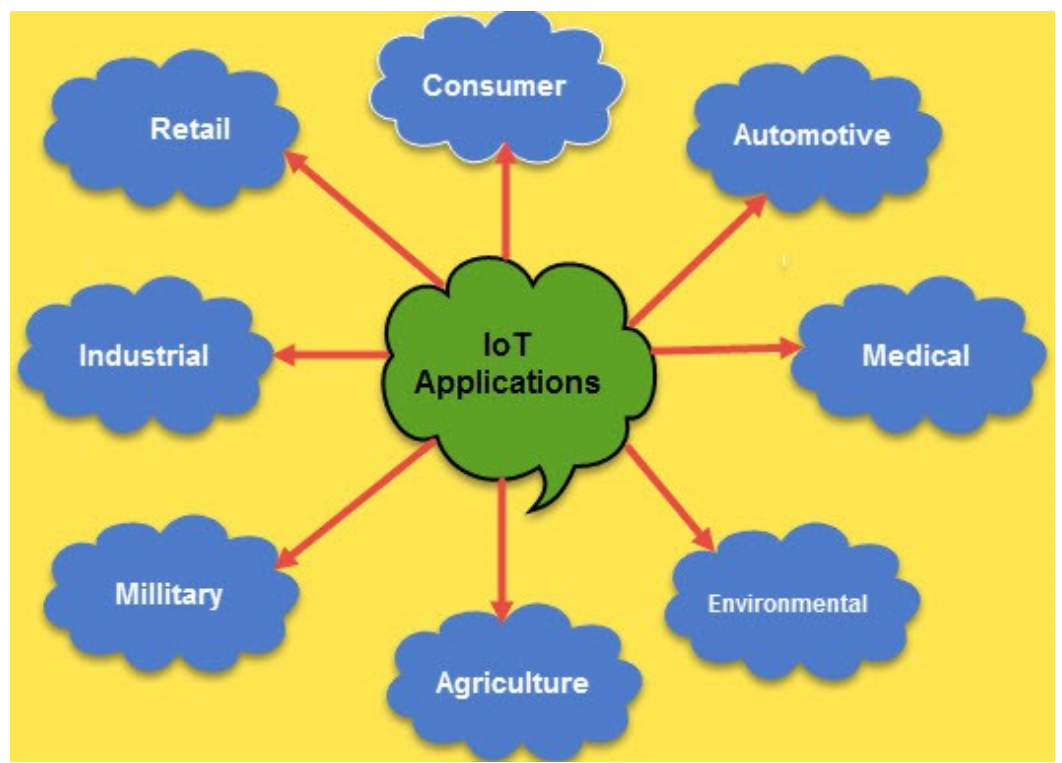

Fig. 3 IoT Applications

\subsection{Smart Living}

In smart living, it consists of remote controlling appliances that are used to switch ON/OFF any device remotely and saves energy. Weather is used for displaying weather conditions such as temperature, humidity, rain levels, speed of the wind. Smart Home Appliances used for the LCD screen on refrigerators tells the items that are present inside and expiry date of the items, ingredients to buy in the future and all the information is available on the app. Figure 3 explains the applications of IoT. Washing machines allows monitoring the laundry remotely by adjusting temperature control and monitors self-cleaning feature

Safety monitoring is used for monitoring cameras, alarm systems at home, and making them feel better. Intrusion Detection System is used for the detection of opening doors to prevent intruders. It is used for monitoring energy and water consumption at home to save costs and resources.

\subsection{Smart Agriculture}

Smart agriculture consists of greenhouse controls and the micro-climate to increase the manufacturing of fruits and vegetables. Compost is used to monitor the levels of humidity and temperature to avoid microbial and other pollutants. Animal Tracking is used to locate and identifies animals grazing in open fields and it also studies air quality and ventilation on farms. Offspring Care is used in animal farms to monitor the growing conditions of the offspring and ensures the plant's survival and health. Field Monitoring reduces spoilage and plant waste by frequent monitoring of accurate data collection and management of fields.

\subsection{Smart Parking}

Smart Parking is used to monitor the available city parking spaces and making the user identify the nearest available space for parking. Waste Management enables the detection of garbage levels in the containers so that they can optimize routes for waste collection. Garbage cans are installed with RFID tags which inform the worker to indicate when the garbage has been thrown off.

\subsection{Smart Cities}

In smart cities, it consists of structural health used for monitoring the material conditions of buildings and bridges. It also monitors if any vibrations are happening in the buildings (Gabhane et al. 2017). Lighting gives smart and weathers adaptive lighting in street lights. Safety consists of control of fire, emergency warning services, digital video monitoring. Transportation consists of smart roads and highways with warning messages and diversions for any unexpected events. 
Journal of ISMAC (2020)

Vol.02/ No.04

Pages: $181-189$

http://irojournals.com/iroismac/

DOI: https://doi.org/10.36548/jismac.2020.4.001

\subsection{Smart Industry}

The smart industry consists of explosive and toxic gases consists of detection of gas level and leakage in industry, monitoring of toxic gas, and level of oxygen inside chemical plants and it also monitors the level of oil and gas in storage tanks and pipelines. In a smart industry, Maintenance and Repair include early detection of any malfunction units, and service maintenance can be automatically assigned for a particular failure.

\subsection{Smart Environment}

In a smart environment, it consists of air pollution monitoring which controls $\mathrm{CO}_{2}$ emission from factories and pollution emitted by cars. Forest Fire Detection is used for gas monitoring and to make alert zones. Weather monitoring includes humidity, temperature, pressure, early detection of an earthquake. Water Quality checks the quality of water for drinking and the study of water suitability in rivers. River Floods are used for monitoring the level of water in the dam and reservoirs during rainy time.

\subsection{Smart Energy}

In smart energy, it consists of a smart grid that describes monitoring and managing the energy consumption in the industries. Wind Powerhouse is used to monitor the energy flow from wind turbines and powerhouse and it also provides bidirectional communication with customers. Then, Smart meters are used to analyze consumption patterns. All the Power Supply Controllers has controllers for AC-DC power supply related to consumer electronic applications and telecommunications. Photovoltaic Installations are used to monitors and optimize the performance in solar energy plants.

\section{Latest challenges and trends of IoT}

The IoT smart systems for the new generation were suggested by Kinza Shafique et al. based on the latest challenges, trends, and emerging scenarios. The proposed research presents a comprehensive and substantial overview of fifth-generation IoT due to increased rates of data requiring both edge and cloud computing platforms (Shafique et al. 2020). Those cellular networks furnish crucial technologies for IoT technology development. The management of a large amount of big data in IoT from all the junctions of the network is a difficult chore and the data centers concentrating on energy efficiency are mainly contemplated. To carry through these problems, AI algorithms, machine learning techniques, and artificial neural networks need to be positioned for energy regulation and making automated decisions. Most of the applications are befit for the Internet of Things possessing traffics like elastic and in-elastic. The developer concentrates on implementation that is detain unbiased which comprises scheduling and observing applications (Perera et al. 2013). The responsive example from the network for inelastic traffic is video streaming. Dually the traffics provides their key entertainment with increased quality of service must be satisfied by IoT. It even enlarges gear means utilization of System configuration and Independent protection layers. The architecture feature is centered on swapping energy-based information between these layers and it reduces the time duration of resting sensor nodes. The sensor nodes function in two ways such as periodic and trigger and also supervised by gateway stations which are further overseen by base stations.

The variation coefficient is dignified allying the post and pre values. The received data cannot be varied either latest or old only the rest time duration can lengthen or shorten. The transmission betwixt the cloud resources and sensor nodes frame-up by e nodes relying on their distant and battery extent which are granted according to needs (Bruneo et al. 2019). Using the platform of cloud computing, the processing layer restores, directs, inspects the data through the resource issuer. This issuer of resources mainly for data processing in a sequential manner by awarding the hardware resources based on the requirements of the data analyzer, control, and sensing layer. Eventually, the transceiver considers the full-duplex also intervenes in the time division and frequency division duplexing. These processes can be accomplished by making use of silicon transistors which acts as speedy switches and permits to broadcast on the alike frequency. These network structures are built to tactile the fatten traffic, introduction of new junctions, network environment efficiency, and flexibility of network expansion (Ammar et al. 2018). The standard process of $5^{\text {th }}$ Generation IoT has two types are dealing with technology, wireless concerns and data processing, and privacy and security policies of data. 
Journal of ISMAC (2020)

Vol.02/ No.04

Pages: 181-189

http://irojournals.com/iroismac/

DOI: https://doi.org/10.36548/jismac.2020.4.001

\section{IoT Smart Home Architecture}

The IoT architecture implementation for smart home was presented by Ganesh et al. 2017 using GSM technology. This proposed framework authorizes the users to manage and control the smart objects using an internet connection. Figure 4 explains the architecture of IoT smart home (Ganesh et al. 2017). Using a Global system for mobile communication and technology of the internet fabricates a hook-up between the smart home and its users or from the web server develops wireless communication-based GSM. The outline of architecture is done by web by giving like SMS messages which are transfigured from the inputs of the user and finally the commands are sent to the network of GSM which are implemented to sway electronic gadgets. The authorized users can dispatch commands to web devices that are connected to the network and the data of users stay in the condensed database through the application programming interface.

The GSM communication is the initial technology used for communication, the IoT representative plays the major architecture part so it copes server information, module interplays, and learning post citations. The element of the GSM receiver behaves as an entry for the transmission module. The module brain is known as a microcontroller, its jurisdictions complete devices personally in the smart home. Avail oneself of GSM SMS for data assembling and transference and proficient to detect and benchmark the devices in the located environment by the pattern for several merits like instant delivery, protected data loss, minimum cost, and energy coherent. IoT is the unit of hardware and software that ceaselessly tracks the webserver and module of GSM from the smart application.

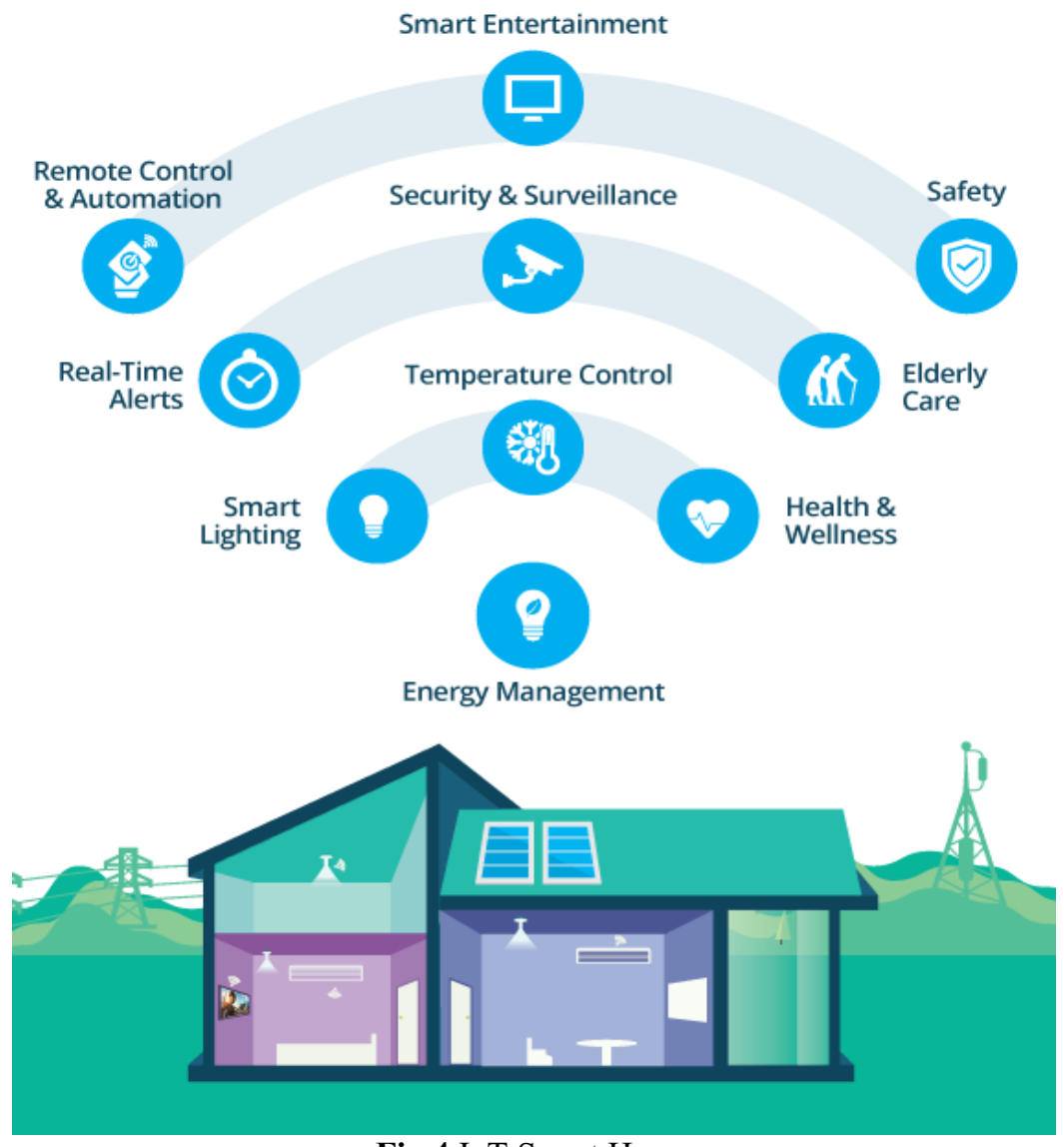

Fig.4 IoT Smart Home

The recent issues, solutions, and directions of the smart home system using IoT were advanced by Gabhane et al. The meteoric technology growth and refinement in methodology emerge different snags that directs how to manage the entire system, server security, and smart home security (Bhavna et al. 2018). The collection 
of data is processed by the sensing layer, transfer it to the network layer which wields the internet for passing the information to another layer for incompatible purposes. The security problem arises when sundry nodes are connected to the internet. The establishment of the first-period key shared between the sensor nodes in wireless and superintend the center is the leading obstacle of the network. The graphical interface of the user is illuminated by the received data from the smart apparatus. The structure of the network should be fashioned in a manner that every object linked to it would organize uniquely.

\section{Home Automation}

The home automation smart system was suggested by Bhavna et al. using the Internet of Things (Gubbi et al. 2013). The proposed arrangement is the appliance automation within our environment which allows us to control and manage even in critical conditions. The communication between the entities provisions by enabling different technologies. In a wireless connection, IoT enhances the webbing for collecting and analyzing the data from devices then resends it to computers or mobiles. Yearly concerns IoT has developed in the field of information technology and communication technology. The principal requirements of the security automation system are data theft, intrusion, and so on. It paves a way for objects to be remotely managed and sensed across the network and results in attaining more benefits. In some applications, the IoT is coupled with actuators and sensors where the technology flatters an occurrence of the most familiar class called cyber-physical systems, which includes various automation technologies like smart homes, smart cities, etc. It can be able to interoperate with the internet underpinning. A link or an interface acts between the customer and the device through which the device is operated. The home automation is considered as an asset which makes the infrastructure self-moving. Using these devices of automation controlling activity and other electrical devices can be trapped. The concerned devices can be co-related with the IR receiver to control other objects and even support for its communication. With the help of IR commands more frameworks and actions can be generated to qualify a large number for device controls that solitary the power vent. Improvements can be done with the programming of the computer also. It should be user friendly and should provide authentication protection for security causes. The automation can also flourish it webbased for easy remote control.

\section{Smart Cities}

The service ecosystem for smart cities using IoT was initiated by Dario Bruneo et al. To consummate the smart city's perception, the Internet of Things, Big data, cloud computing, and so on are mooted together to obtain the retorts to solve issues related to it. In modern days the cities are well developed with technological entities, with internet connectivity and drawn more modish in every aspect (Bruneo et al. 2019). The main aim of this research to accomplish a kindred ecosphere, open access IT infrastructure in the urban area which compressed sensors, actuators, other data, and network capitals using legion applications. Those smart city environments include devices which are either public or private based on their inquiries. To boot the infrastructure layer is used where the web resources and allied problems are scrutinized besides amenities of data processing and depository are apt to perform its obligations (Chen et al. 2017). In an unlatched fashion, information to be trucked out of discrete overhauls and contributors mutually inspect to extricate upgraded data and trip elegant gestures. The contextualization constitutes a way to adjust the node behavior below deliberation to the work at that time.

The development of a smart city based on IoT was lodged by M. Mazhar Rathore et al. using Big- data analytical approach. The absolute architecture which is proposed and its implementation are undertaken wield spark atop Hadoop processing framework in realistic surroundings. The features of actions the system implements are arrived by step manner, they are generation, aggregation, collection, filtration, pre-processing, and so forth at the making of resolves. Figure 5 depicts the IoT applications for smart cities. The practical enactment is drawn to thrive in smart cities by a variety of sources of data with enhanced smart systems (Rathore et al. 2016). The fitter system evaluation is more climbable and coherent. The primary conviction is to gain accurate data on a precise device at an exact place at perfect duration to coerce related determination in easy and swift ways. The system proposed achieves security by continual observation of the entire city. The model architecture for analysis and implementation hinged on the requirements of smart areas to organize the analytical process at actual time oblige verdicts. 


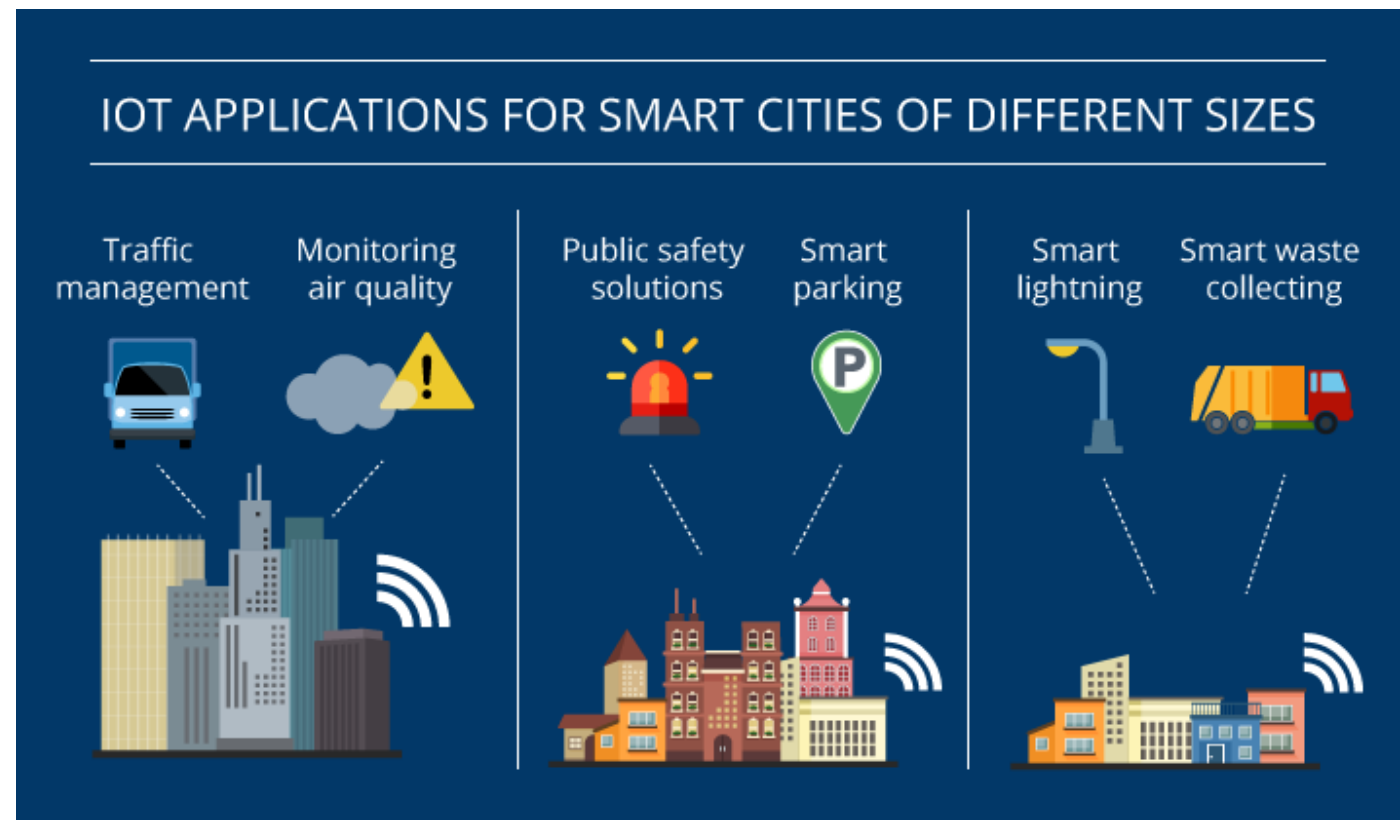

Fig.5 IoT Smart Cities

The offered system is for defining smart digital cities using urban data in real-time by M. Mazhar Rathore et al. for exploiting IoT and Big data. The interrelationship of different IoT objects to cumulate the data of urban areas with the aid of the Internet to instigate a smart digital city. Its services are elongate by introducing a system for transport which is also smart for yielding timely updates and alerts. The system on whole presents a prototype for execution using Hadoop. The challenging task is to heap the data collected by making use of the physical system of cyber and smart devices of IoT for enjoyers and sovereignty findings to effectuate the stipulation of occupants geared toward spruce digital city (Rathore et al. 2017). The chief scheme of this procedure to grasp valid information of the city in a virtuous situation at a specified duration to carry out solutions. It also smoothens the natives in a way prompt.

\section{Conclusion}

Internet of things is one of the major researches thrust in recent days as the applications are not limited into certain domains. Due to the diverse features IoT is widely adopted and a summary of various IoT systems are discussed in this research work to obtain prior knowledge. The analysis is performed based on domain, applications, environment parameters and the facts are discussed with its advantages. Based on the analysis it could be useful for beginning researchers to get influenced over IoT technologies in future for their research works.

\section{References}

1. Ganesh, E. N. (2017). Implementation of IoT architecture for SMART HOME using GSM technology. International Journal of Computer Techniques, 4(1), 42-48.

2. Shafique, K., Khawaja, B. A., Sabir, F., Qazi, S., \& Mustaqim, M. (2020). Internet of things (IoT) for next-generation smart systems: A review of current challenges, future trends and prospects for emerging 5G-IoT scenarios. IEEE Access, 8, 23022-23040.

3. Gabhane, M. J. P., Thakare, M. S., \& Craig, M. M. (2017). Smart Homes System Using Internet-ofThings: Issues, Solutions and Recent Research Directions. International Research Journal of Engineering and Technology, 4(5): 1965 - 1969.

4. Sharma, V., \& Tiwari, R. (2016). A review paper on "IoT" \& It's Smart Applications. International Journal of Science, Engineering and Technology Research, 5(2): 472-476. 
Journal of ISMAC (2020)

Vol.02/ No.04

Pages: $181-189$

http://irojournals.com/iroismac/

DOI: https://doi.org/10.36548/jismac.2020.4.001

5. Bhavna, D., \& Sharma, N. (2018). Smart Home Automation using IoT. International Journal of Engineering Sciences and research technology. 7(5): $435-437$.

6. Vandana, S., \& Ravi, T. (2016). A review paper on "IoT" \& It's Smart Applications. International Journal of Science, Engineering and Technology Research, 5(2): 472 - 476.

7. Zhai, S. L., Zhao, D. S., Wang, Z., \& Zhang, Y. (2012). Research of communication technology on IoT for high-voltage transmission line. International Journal of Smart Grid and Clean Energy, 1(1): 85-90.

8. Yashiro, T., Kobayashi, S., Koshizuka, N. and Sakamura, K. (2013) An Internet of Things (IoT) architecture for embedded appliances, 2013 IEEE Region 10 Humanitarian Technology Conference, Sendai, 2013, 314-319, doi: 10.1109/R10-HTC.2013.6669062

9. Singh, D., Tripathi, G., \& Jara, A. J. (2014). A survey of Internet-of-Things: Future vision, architecture, challenges and services. In 2014 IEEE world forum on Internet of Things (WF-IoT), 287-292.

10. Wang, M., Zhang, G., Zhang, C., Zhang, J., \& Li, C. (2013) An IoT-based appliance control system for smart homes. In 2013 fourth international conference on intelligent control and information processing, pp. 744-747.

11. Soliman, M., Abiodun, T., Hamouda, T., Zhou, J., \& Lung, C. H. (2013, December). Smart home: Integrating internet of things with web services and cloud computing. In 2013 IEEE 5th international conference on cloud computing technology and science, 2: 317-320.

12. Rathore, M. M., Ahmad, A., \& Paul, A. (2016, October). IoT-based smart city development using big data analytical approach. In 2016 IEEE international conference on Automatica (ICA-ACCA), 1-8.

13. Rathore, M. M., Paul, A., Ahmad, A., \& Jeon, G. (2017). IoT-based big data: from smart city towards next generation super city planning. International Journal on Semantic Web and Information Systems (IJSWIS), 13(1), 28-47.

14. Chen, L. J., Ho, Y. H., Lee, H. C., Wu, H. C., Liu, H. M., Hsieh, H. H., ... \& Lung, S. C. C. (2017). An open framework for participatory PM2. 5 monitoring in smart cities. IEEE Access, 5: 14441-14454.

15. Perera, C., Zaslavsky, A., Christen, P., \& Georgakopoulos, D. (2013). Context aware computing for the internet of things: A survey. IEEE communications surveys \& tutorials, 16(1): 414-454.

16. Bruneo, D., Distefano, S., Giacobbe, M., Minnolo, A. L., Longo, F., Merlino, G., ... \& Puliafito, C. (2019). An IoT service ecosystem for smart cities: The Smartme project. Internet of Things, 5: 12-33.

17. Lee, I., \& Lee, K. (2015). The Internet of Things (IoT): Applications, investments, and challenges for enterprises. Business Horizons, 58(4): 431-440.

18. Patel, K. K., \& Patel, S. M. (2016). Internet of things-IOT: definition, characteristics, architecture, enabling technologies, application \& future challenges. International journal of engineering science and computing, 6(5): $6122-6131$.

19. Gubbi, J., Buyya, R., Marusic, S., \& Palaniswami, M. (2013). Internet of Things (IoT): A vision, architectural elements, and future directions. Future generation computer systems, 29(7): 1645-1660.

20. Ammar, M., Russello, G., \& Crispo, B. (2018). Internet of Things: A survey on the security of IoT frameworks. Journal of Information Security and Applications, 38: 8-27. 